\section{Christine Keller Sallenbach}

Der Dachverband Komplementärmedizin (Dakomed) hat 5 Jahre nach der Volksabstimmung über den Verfassungsartikel 118a Komplementärmedizin eine repräsentative Umfrage bei 1001 Stimmberechtigten zur Nutzung, Verankerung und Ausübung sowie zum Image der Komplementärmedizin in Auftrag gegeben.

Die Komplementärmedizin ist heute in der öffentlichen Diskussion weniger präsent, als dies vor und während der Abstimmung vor 5 Jahren der Fall war. Die Zustimmung zum Verfassungsartikel fiel 2009 mit 66\% sehr deutlich aus.

\section{Nutzung von \\ Komplementärmedizin}

Ein Vergleich der Nutzung von Komplementärmedizin in den letzten 7 Jahren zeigt eine klare Zunahme (Abb. 1). Komplementärmedizin zu nutzen hat heute eine grössere Akzep$\operatorname{tanz}$ und scheint eine Selbstverständ-

\title{
Komplementärmedizin weiter im Aufwind
}

Die Nachfrage nach Komplementärmedizin ist in den letzten Jahren weiter gestiegen, und die Bevölkerung ist mehrheitlich von der Wirksamkeit und Seriosität der Komplementärmedizin überzeugt. Handlungsbedarf gibt es insbesondere bei der Verankerung der Komplementärmedizin an den Universitäten.

lichkeit zu sein. Auch die Bekanntheit von Komplementärmedizin und diesbezüglichen Therapieangeboten scheint grösser zu sein, wie insbesondere die starke Nutzungszunahme bei Therapeutenbesuchen und bei der Selbstmedikation vermuten lassen.

\section{Verankerung der Komplementärmedizin}

Die Mehrheit der Befragten unterstützt eine stärkere Verankerung der Komplementärmedizin im Schweizerischen Gesundheitswesen (Abb. 2). $62 \%$ der Befragten befürworten die Aufnahme der ärztlichen Komplementärmedizin in die Grundversicherung, 22\% haben dazu keine Meinung, und $16 \%$ lehnen dies ab. Ebenso wünscht eine klare Mehrheit von 57\% der Bevölkerung, dass Ärzte und Apotheker bereits im Studium Grundkenntnisse über Komplementärmedizin erlernen sollen, und gar $67 \%$ der Befragten sind für die Erforschung der Komplementärmedizin an den Universitäten.

Ein Drittel der Befragten ist zudem der Ansicht, dass sich Bund und Kantone nicht genügend für die Berücksichtigung der Komplementärmedizin im Schweizerischen Gesundheitswesen einsetzen. Es ist deshalb weiterhin die Aufgabe des Dakomed, Bund und Kantonen auf die Finger zu schauen und den politischen Druck zur Berücksichtigung der Komplementärmedizin im Schweizerischen Gesundheitswesen aufrechtzuerhalten.

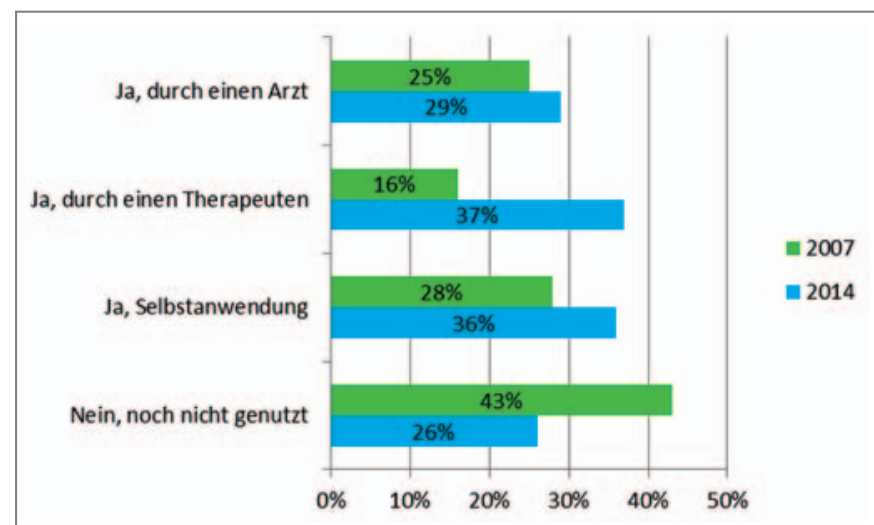

Abb. 1. Antworten auf die Frage: «Nutzen Sie bzw. haben Sie selber schon Komplementärmedizin (Alternativmedizin) genutzt (z.B. pflanzliche Arzneimittel, Chinesische Medizin, Homöopathie, Akupunktur usw.)?»

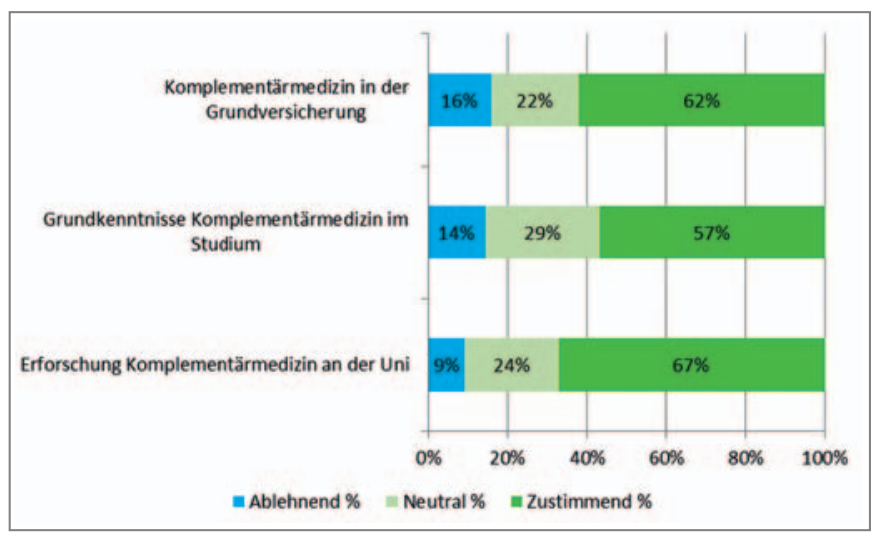

Abb. 2. Antworten zur Verankerung der Komplementärmedizin in der Grundversicherung.

\section{KARGER}

Fax +497614520714 Information@Karger.com www.karger.com
(๑) 2014 S. Karger GmbH, Freiburg

Accessible online at: www.karger.com/sz
Christine Keller Sallenbach

Geschäftsführerin Dachverband Komplementärmedizin (Dakomed)

Amthausgasse 18, 3011 Bern, Schweiz

christine.keller@dakomed.ch www.dakomed.ch 


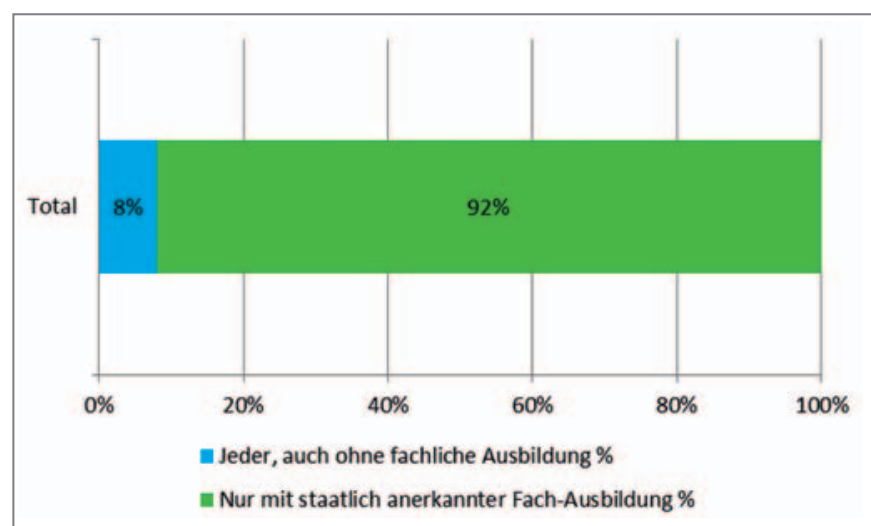

Abb. 3. Ausübung der nichtärztlichen Komplementärmedizin.

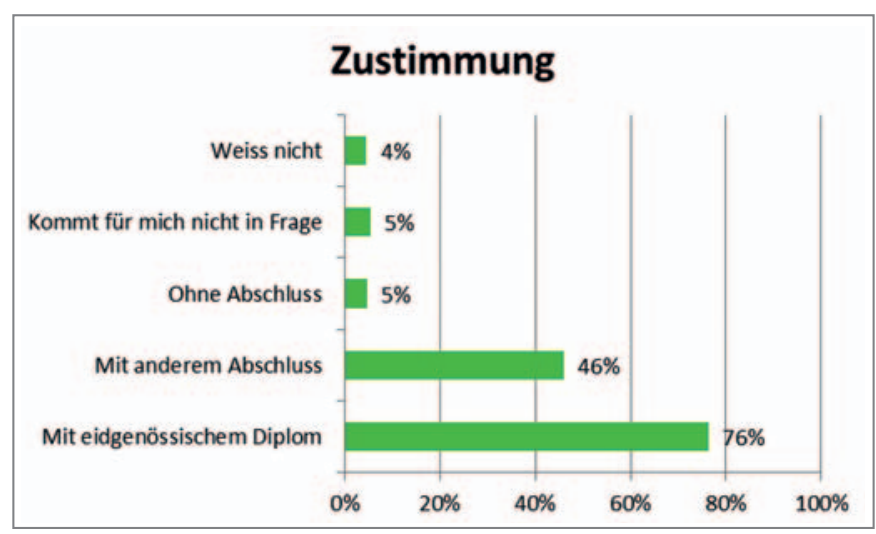

Abb. 4. «Wer kommt als Komplementärtherapeut infrage?»

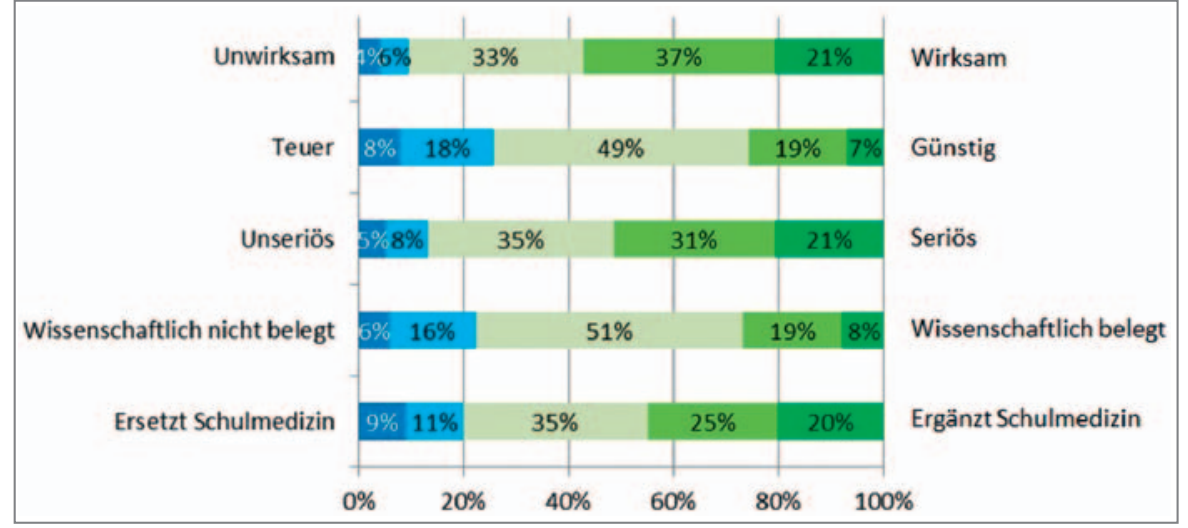

\section{Qualität der \\ Komplementärmedizin}

Die Bevölkerung ist klar der Meinung, dass für die Berufsausübung als Naturheilpraktiker ein nationales Diplom notwendig ist; ebenso bevorzugt eine Mehrheit der Bevölkerung, dass Komplementärtherapeuten über ein eidgenössisches oder ein anderes Diplom verfügen (Abb. 3, 4). Damit widerspricht die Bevölkerung der Liberalisierungstendenz einiger Kantone, welche die Berufsausübung von Naturheilpraktikern und Komplementärtherapeuten vollständig freigegeben haben.

\section{Image der \\ Komplementärmedizin}

Das Image der Komplementärmedizin ist bei der Bevölkerung gut. Sie gilt als Ergänzung der Schulmedizin sowie als wirksam und seriös. Den wissenschaftlich belegten Nutzen von Komplementärmedizin gilt es zu verbessern (Abb. 5).

\section{Fazit}

Die Akzeptanz und das Wissen über Komplementärmedizin sind in der Schweiz gut. Komplementärmedizin gilt als wirksam, seriös und die Schulmedizin ergänzend. Nichtsdestotrotz geht dem Dakomed die Arbeit nicht aus. Sein Fokus für die nächsten Jahre muss insbesondere auf der Stärkung des Qualitätsbewusstseins in den Kantonen und der stärkeren Verankerung der Komplementärmedizin in den Universitäten liegen - dies sowohl in der Lehre als auch in der Forschung. Mit aktuell je einem Institut für Komplementärmedizin an den
Abb. 5. Image der Komplementärmedizin bei der Bevölkerung.
Universitäten Bern und Zürich, einer Gruppe für Lehre und Forschung in Lausanne sowie einer Fachstelle für Phytopharmazie an der Zürcher Hochschule für Angewandte Wissenschaften ist sie im Vergleich zu den mehr als 1000 Professuren für Schulmedizin deutlich untervertreten.

Die vollständige Bevölkerungsbefragung finden Sie unter: $w w w . d a k o-$ med.ch/publikationen. 